\section{RMD Open}

Rheumatic \& Musculoskeletal Diseases

\title{
Role of targeted therapies in rheumatic patients on COVID-19 outcomes: results from the COVIDSER study
}

Jose María Álvaro Gracia, ${ }^{1}$ Carlos Sanchez-Piedra, ${ }^{2}$ Javier Manero, ${ }^{3}$

María Ester Ruiz-Lucea, ${ }^{4}$ Laura López-Vives, ${ }^{5}$ Cristina Bohorquez, ${ }^{6}$ Julia Martinez-Barrio, ${ }^{1}$ Gema Bonilla, ${ }^{7}$ Paloma Vela, ${ }^{8}$

María Jesús García-Villanueva, ${ }^{9}$ María Teresa Navío-Marco, ${ }^{10}$ Marina Pavía, ${ }^{11}$ María Galindo, ${ }^{12}$ Celia Erausquin, ${ }^{13}$ Miguel A Gonzalez-Gay (D) ,14 Inigo Rua-Figueroa, ${ }^{13}$ Jose M Pego-Reigosa, ${ }^{15}$ Isabel Castrejon, ${ }^{1}$ Jesús T Sanchez-Costa, ${ }^{2}$ Enrique González-Dávila, ${ }^{16}$ Federico Diaz-Gonzalez (D) , ${ }^{17,18}$ on behalf of the COVIDSER study group

To cite: Álvaro Gracia JM, Sanchez-Piedra C, Manero J, et al. Role of targeted therapies in rheumatic patients on COVID-19 outcomes: results from the COVIDSER study. RMD Open 2021;7:e001925. doi:10.1136/ rmdopen-2021-001925

- Additional supplemental material is published online only. To view, please visit the journal online (http://dx.doi.org/10. 1136/rmdopen-2021-001925).

Received 9 September 2021 Accepted 9 November 2021

Check for updates

(c) Author(s) (or their employer(s)) 2021. Re-use permitted under CC BY-NC. No commercial re-use. See rights and permissions. Published by BMJ.

For numbered affiliations see end of article.

Correspondence to Dr Federico Diaz-Gonzalez; federico.diaz.gonzalez@gmail. com

\section{ABSTRACT}

Objectives To analyse the effect of targeted therapies, either biological (b) disease-modifying antirheumatic drugs (DMARDs), targeted synthetic (ts) DMARDs and other factors (demographics, comorbidities or COVID-19 symptoms) on the risk of COVID-19 related hospitalisation in patients with inflammatory rheumatic diseases.

Methods The COVIDSER study is an observational cohort including 7782 patients with inflammatory rheumatic diseases. Multivariable logistic regression was used to estimate ORs and $95 \%$ Cls of hospitalisation. Antirheumatic medication taken immediately prior to infection, demographic characteristics, rheumatic disease diagnosis, comorbidities and COVID-19 symptoms were analysed.

Results A total of 426 cases of symptomatic COVID-19 from 1 March 2020 to 13 April 2021 were included in the analyses: 106 (24.9\%) were hospitalised and 19 (4.4\%) died. In multivariate-adjusted models, bDMARDs and tsDMARDs in combination were not associated with hospitalisation compared with conventional synthetic DMARDs (OR $0.55,95 \% \mathrm{Cl} 0.24$ to 1.25 of b/tsDMARDs, $\mathrm{p}=0.15$ ). Tumour necrosis factor inhibitors (TNF-i) were associated with a reduced likelihood of hospitalisation (OR $0.32,95 \% \mathrm{Cl} 0.12$ to $0.82, \mathrm{p}=0.018$ ), whereas rituximab showed a tendency to an increased risk of hospitalisation (OR 4.85, 95\% $\mathrm{Cl} 0.86$ to 27.2). Glucocorticoid use was not associated with hospitalisation (OR 1.69, 95\% Cl 0.81 to 3.55). A mix of sociodemographic factors, comorbidities and COVID-19 symptoms contribute to patients' hospitalisation.

Conclusions The use of targeted therapies as a group is not associated with COVID-19 severity, except for rituximab, which shows a trend towards an increased risk of hospitalisation, while TNF-i was associated with decreased odds of hospitalisation in patients with rheumatic disease. Other factors like age, male gender, comorbidities and COVID-19 symptoms do play a role.

\section{Key messages}

What is already known about this subject?

- The potential effect of targeted therapies on the prognosis of COVID-19 in rheumatic patients remains to be clarified with studies yielding inconsistent results.

What does this study add?

- This study conducted in a structured cohort of rheumatic patients shows that targeted therapies are not associated with a different prognosis of COVID-19, except for rituximab, linked with an increased risk of hospitalisation, and tumour necrosis factor inhibitors, associated with a reduction in that risk.

- Some COVID-19 symptoms like asthaenia, nausea, fever and dyspnoea were associated to an increased risk of hospitalisation.

How might this impact on clinical practice or further developments??

- Our results support that rheumatic patients treated with targeted therapies do not require special attention for COVID-19, except those treated with rituximab, who are at risk for more severe disease. Some COVID-19 symptoms could guide in the risk stratification of rheumatic patients who develop the infection.

\section{INTRODUCTION}

Since the outbreak of the COVID-19 pandemic, the question on the clinical course of patients with rheumatic and musculosketal diseases (RMDs) receiving targeted therapies, either biological disease-modifying antirheumatic drugs (bDMARDs) or targeted synthetic DMARDs (tsDMARDs) has been a matter of discussion. On one hand, patients with RMDs on 
targeted therapies are known to have an increased risk for severe bacterial and viral infections. ${ }^{12}$ On the contrary, some of these drugs, such as interleukin-1, interleukin-6 or Janus kinase (JAK) inhibitors, could have a protective role on the cytokine storm syndromes involved in the physiopathology of severe COVID-19 and, in fact, have been studied as potential treatments of severe COVID-19. ${ }^{3-5}$

The information on the severity of the clinical course of COVID-19 (ie, hospitalisation or death risk) in patients with RMDs is confusing because of contradictory results and potential confounders in the various studies published so far, with suggestions of a similar course to that of the general population, a worse prognosis or even a better one in patients with some conditions like systemic autoimmune diseases. ${ }^{6-13}$

The potential effect of ts/bDMARDs on the prognosis of these patients is also poorly understood with studies showing no effect, worsening or even improving the outcome of these patients. ${ }^{89-19}$ It is important to clarify this to adequately inform therapeutical decisions in patients who develop COVID-19 while receiving b/tsDMARDs. Some of the possible reasons for this heterogeneity of previous studies rely on the race for rapid generation and analysis of data in the context of the pandemic.

This report analyses COVID-19 prognosis (assessed by risk of COVID-19 related hospitalisation) in patients with RMDs receiving targeted therapies, either bDMARDs or tsDMARDs belonging to three well-established Spanish databases included in the COVIDSER study.

\section{PATIENTS AND METHODS}

\section{Study design and data sources}

The COVIDSER study is an observational cohort of patients from three Spanish databases of patients with RMDs: BIOBADASER III, CARdiovascular in
rheuMAtology (CARMA) and RELESSER registries, all promoted by the Spanish Society of Rheumatology (SER). All patients from participant centres in these three registries (ie, 28 sites in the BIOBADASER, 61 sites in the CARMA and 39 sites in the RELESSER-Pros) who had a diagnosis of rheumatoid arthritis (RA), spondyloarthritis (SpA), systemic lupus erythematosus (SLE) and other inflammatory autoimmune rheumatic disease were invited to participate. A total of 7782 patients diagnosed with RA, SpA, SLE and other inflammatory rheumatic diseases were included in the COVIDSER study (see figure 1). The COVIDSER patient group has not been included in other similar studies.

BIOBADASER III is a prospective national registry to evaluate the long-term safety of patients with rheumatic diseases who are undergoing treatment with targeted therapy: bDMARDs, including biosimilars, and tsDMARDs. ${ }^{20}$ The CARMA project is a prospective 10-year follow-up cohort study designed to determine the risk of cardiovascular mortality in patients older than 18 years with RA, axial-spondyloarthritis (AxSpA) or psoriatic arthritis (PsA) ${ }^{21}$ RELESSER-PROS is a prospective study of patients with SLE included in the original RELESSER study. ${ }^{22}$ These registries had been approved by the Spanish Agency of Medicines and Medical Devices as well as by the respective ethics committees of all participant health institutions, and all participants provide their written informed consent to be included in the database and for using their data for investigational purposes. We informed all ethics committees involved in those registries about the project presented herein.

\section{Case definition and study variables}

Patients with a confirmed or suspected diagnosis of COVID-19 included in any of the three registries from 1

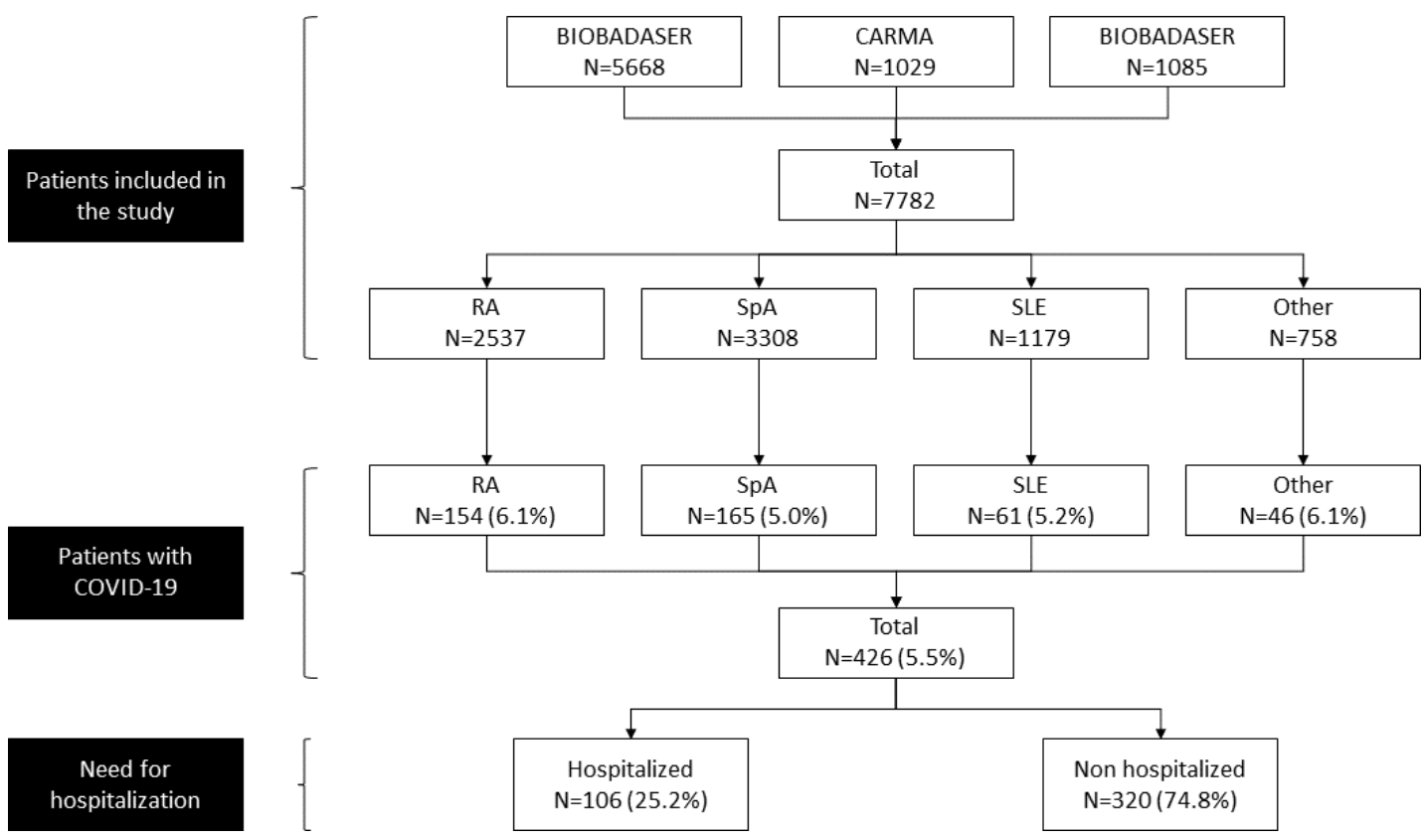

Figure 1 Flo -chart. Patients included in the analysis. 
March 2020 to 13 April 2021 were analysed. We considered that patients were cases of COVID-19 if they had either PCR or antigen test positive (confirmed cases). We also included suspected cases during the first months of the pandemic - due to the limited availability of diagnostic tests-as patients with clinical symptoms/signs highly compatible with COVID-19, namely, fever, dyspnoea, cough and pneumonia, as assessed by X-ray or CT scan and positive epidemiological factors such as close contacts to patients with COVID-19. Cases were identified during the follow-up visits of the corresponding registries and/or revising the clinical records of the patients included in the database.

The following COVID-19 related information was recorded from the identified cases: date of diagnosis, type of case and symptoms of COVID-19, including fever or chills, cough, shortness of breath or difficulty breathing, anosmia, ageusia/dysgeusia, sore throat, arthralgia, myalgias, rhinorrhoea, nausea/vomiting, diarrhoea, fatigue, headache, chest pain and abdominal pain. Hospitalisation, ICU admission and the outcome of the COVID-19 were recorded. Regarding patients and disease characteristics, the following data were collected: age, gender, body mass index (BMI), diagnosis, date of diagnosis, comorbidities (Charlson index) and risk factors (smoking status). Rheumatic disease activity prior to and 3 months after COVID-19 was categorised into remission, mild, moderate or severe activity at the investigator's judgement. Regarding treatment, information on the treatment of rheumatic disease was recorded, categorised as non-biologicalDMARD, including conventional synthetic (cs) DMARD (methotrexate, hydroxychloroquine/chloroquine, others) and tsDMARDs (baricitinib and tofacitinib), bDMARDs (tumour necrosis factor (TNF) inhibitors, anti-IL-1 inhibitor, anti-IL-17 Inhibitor, anti-B cell (anti-CD20 and anti-BAFF), IL-6 inhibitors and others), glucocorticoids and other treatments (namely, non-steroidal anti-inflammatory drugs (NSAIDs) and ACEI) at the time of the onset of the symptoms. The remaining information used in this retrospective study was extracted from the corresponding registry.

\section{Statistical analysis}

Quantitative variables are described with the mean and $\mathrm{SD}$ or the median and IQR, and the qualitative variables with the absolute and relative frequencies. For the bivariate analysis, quantitative variables were compared using the Student's t test or analysis of variance and for non-parametric variables with the Mann-Whitney $\mathrm{U}$ or Kruskal Wallis $\mathrm{H}$ tests, and qualitative variables using the $\chi^{2}$ test or Fisher's exact test. Descriptive analysis was stratified according to the use of ts/bDMARDs.

We evaluated the factors associated with hospitalisation among patients with COVID-19 using a logistic regression model. Regardless of the procedure, the independent variables included in the model were the following: treatment of rheumatic disease categorised as csDMARD, bDMARD and/or tsDMARD, demographics (age, sex, race and tobacco); rheumatic disease diagnosis and disease duration; comorbidities including chronic obstructive pulmonary disease, diabetes, hypertension, BMI, obesity, neoplasia, chronic liver disease and chronic kidney disease; and the symptoms of COVID-19.

Candidate variables were chosen as those that in the univariate study (or bivariate study according to table $3)$ presented a p value $<0.1$. Logistic regression was then applied with the Wald backward variable selection method on all of them (cut-off point 0.10), except for sex, diagnosis of rheumatic disease, type of biological and glucocorticoid use, which were kept as fixed factors (Method enter). Glucocorticoids were forced into the model because of their potential role in promoting infections. The discrimination properties of these models were assessed with the area under the receiver operating characteristic curve (AUROC), as well as the detection rates of hospitalisation and kappa coefficient of concordance between observed and predicted values. All statistical analyses were performed using SPSS (V.25, IBM SPSS). Results were considered significant if $\mathrm{p}<0.05$.

\section{RESULTS}

Patient disposition and characteristics

A total of 7782 patients were included in the study. Patients were diagnosed with SpA (including PsA, AxSpA (including non-radiographic forms), undifferentiated SpA, reactive arthritis, enteropathic arthritis and SAPHOSynovitis, Acne, Pustulosis, Hyperostosis and Osteitis-) $(\mathrm{n}=3308,42.5 \%)$, RA ( $\mathrm{n}=2537,32.6 \%)$, SLE ( $\mathrm{n}=1179$, $15.2 \%$ ), of other rheumatic diseases including, juvenile idiopathic arthritis $(n=130)$, vasculitis $(n=71)$, Behçet's disease $(n=30)$, Sjögren's Syndrome $(n=33)$ and other less common inflammatory rheumatic conditions $(n=758$, $9.7 \%$ ) (figure 1). We identified $426(5.5 \%)$ patients with symptomatic COVID-19 during the study period, with similar proportion among patients with RA 6.1\% (154 out of 2537 patients), SpA 5\% (165 out of 3308 patients), SLE 5.1\% (61 out of 1179 patients) or other rheumatic diseases $6.1 \%$ ( 46 out of 758 patients) ( $\mathrm{p}=0.26$ ).

The demographic and clinical characteristics, comorbidities, as assessed by the Charlson index, and the patients' antirheumatic treatments (grouped by their nature into bDMARDs, tsDMARDs and csDMARDs, glucocorticoids, NSAIDs and ACE inhibitors and bDMARDs also by mechanism of action), globally and by diagnosis, are shown in table 1 . Patients were predominantly middle-aged women $(69 \%)$ with long-standing disease (11.0, IQR: 5.8-17.4 years). The diagnosis of COVID-19 dated from 1 March 2020 to 13 April 2021 and was confirmed by a positive PCR or antigen test in a $69 \%$ $(n=294)$ of the cases $(n=426)$. Most patients, 386 recovered without sequelae $(91.0 \%)$ but $19(4.0 \%)$ died.

Effect of $b$ /tsDMARDs on the risk of hospitalisation

Overall, $106(24.9 \%)$ of the patients with symptomatic COVID-19 were hospitalised. Hospitalisation was more 
Table 1 Characteristics of the patients with rheumatic diseases and symptomatic COVID-19

\begin{tabular}{|c|c|c|c|c|c|}
\hline Variable & RA $(n=154)$ & SpA $(n=165)$ & SLE $(n=61)$ & Other RD ( $n=46)$ & Total $(n=426)$ \\
\hline Age (years), median (IQR) & $59.5(48.1-67.5)$ & $52.8(44.6-60.9)$ & $48.5(40.4-57.9)$ & $57.0(31.1-64.6)$ & $55.1(44.6-63.3)$ \\
\hline Sex (male), n (\%) & $29(19 \%)$ & $87(53 \%)$ & $6(10 \%)$ & $11(24 \%)$ & $133(31 \%)$ \\
\hline Caucasian & $126(82)$ & $160(97)$ & $54(88)$ & $42(92)$ & $382(90)$ \\
\hline Latin American & $22(14)$ & $3(2)$ & $6(10)$ & $2(4)$ & $33(8)$ \\
\hline \multicolumn{6}{|l|}{ Tobacco, n (\%) } \\
\hline Current smoker & $15(10)$ & $23(14)$ & $10(16)$ & $3(7)$ & $51(12)$ \\
\hline Former smoker & $35(23)$ & $38(23)$ & $25(41)$ & $6(13)$ & $104(24)$ \\
\hline Non-smoker & $104(67)$ & $104(63)$ & $26(43)$ & $37(89)$ & $271(64)$ \\
\hline BMI, mean $\pm S D$ & $27.5 \pm 5.1$ & $28.5 \pm 5.4$ & $26.3 \pm 6.0$ & $25.5 \pm 4.7$ & $27.5 \pm 5.4$ \\
\hline \multicolumn{6}{|l|}{ COVID-19 diagnosis, $\mathrm{n}(\%)$} \\
\hline Confirmed cases & $113(73)$ & $111(67)$ & $38(62)$ & $32(70)$ & $294(69)$ \\
\hline Suspected cases & $41(27)$ & $54(33)$ & $23(38)$ & $14(30)$ & $132(31)$ \\
\hline \multicolumn{6}{|l|}{ COVID-19 outcomes, n (\%) } \\
\hline Recovered without sequelae & $136(88)$ & $156(94)$ & $54(88)$ & $40(87)$ & $386(91)$ \\
\hline Recovered with sequelae & $9(6)$ & $6(4)$ & $3(5)$ & $3(7)$ & $21(5)$ \\
\hline Death & $9(6)$ & $3(2)$ & $4(7)$ & $3(7)$ & $19(4)$ \\
\hline
\end{tabular}

Treatments (last treatment previous to COVID-19 diagnosis) bDMARD, n (\%)

\begin{tabular}{|c|c|c|c|c|c|}
\hline TNF inhibitors & $54(35)$ & $105(64)$ & - & $16(35)$ & $175(41)$ \\
\hline IL-17 inhibitors & - & $18(11)$ & - & - & $18(4)$ \\
\hline IL-1-inhibitor & $2(1)$ & - & - & - & $2(0.5)$ \\
\hline Anti-CD20 & $9(6)$ & - & $3(5)$ & $3(7)$ & $15(3.5)$ \\
\hline Anti-BAFF & - & - & $9(15)$ & - & $9(2)$ \\
\hline $\begin{array}{l}\text { Others (T cell activation } \\
\text { inhibitor, IL-12/23 inhibitor) }\end{array}$ & $13(8)$ & $8(5)$ & $1(2)$ & $5(11)$ & $27(6)$ \\
\hline \multicolumn{6}{|l|}{ tsDMARD, n (\%) } \\
\hline Apremilast (inh. PDE4) & - & $7(4)$ & - & - & $7(2)$ \\
\hline Baricitinib & $15(10)$ & $1(0.6)$ & - & - & $16(4)$ \\
\hline Chloroquine & $11(7)$ & - & $32(53)$ & $3(7)$ & $46(11)$ \\
\hline Other & 27 (17) & $15(9)$ & $9(15)$ & $3(7)$ & $54(13)$ \\
\hline Glucocorticoids, n (\%) & $81(53)$ & $21(13)$ & $31(51)$ & $18(39)$ & $151(35)$ \\
\hline
\end{tabular}




\begin{tabular}{|c|c|c|c|c|c|}
\hline Variable & RA ( $n=154)$ & SpA $(n=165)$ & SLE $(n=61)$ & Other RD $(n=46)$ & Total $(n=426)$ \\
\hline NSAIDs, n (\%) & $36(23)$ & $58(35)$ & $4(7)$ & $4(9)$ & $102(24)$ \\
\hline ACEI, n (\%) & $16(10)$ & 17 (10) & $10(16)$ & $2(4)$ & $45(11)$ \\
\hline \multicolumn{6}{|l|}{ Comorbidities } \\
\hline \multicolumn{6}{|l|}{ Charlson index } \\
\hline Mean \pm SD & $2.2 \pm 1.8$ & $1.7 \pm 1.0$ & $2.3 \pm 1.9$ & $2.5 \pm 2.2$ & $2.1 \pm 1.6$ \\
\hline Median (IQR) & $2(1-3)$ & $1(1-2)$ & $1(1-3)$ & $1.5(1-4)$ & $1(1-3)$ \\
\hline
\end{tabular}

ACEI, ACE inhibitors; bDMARD, biological disease-modifying antirheumatic drugs; BMI, body mass index; csDMARD, conventional synthetic disease-modifying antirheumatic drug; DAS-28, Disease activity score-28; ICU, intensive care unit; IL, interleukin; NSAIDs, non-steroidal anti-inflammatory drugs; RA, rheumatoid arthritis; RD, rheumatic diseases; SLE, systemic lupus erythematosus; SpA, spondyloarthropathies; TNF, tumour necrosis factor; tsDMARD, targeted synthetic disease-modifying antirheumatic drugs.

frequent among patients with RA (54 out of 154 patients, $35.1 \%)$ and less frequent among SLE (13 out of 48 patients, $21.3 \%$ ) and SpA (30 out of 165 patients, $18.2 \%)$ patients $(\mathrm{p}=0.0018)$. Admission to the intensive care unit (ICU) was required by 21 patients $(4.9 \%$ ) (table 1 ).

Table 2 shows the demographic characteristics, COVID-19 symptoms, rheumatic disease and comorbidities of the studied population according to the use of ts/bDMARDs or csDMARDs prior to COVID-19 diagnosis and to the outcome of requiring hospitalisation or not. Among hospitalised patients $(\mathrm{n}=106)$, a total of $71(67.0 \%)$ were receiving ts/bDMARDs and 35 (33\%) csDMARDs. However, the proportions of patients that required hospitalisation were similar between both treatment groups, 23\% (71 out of 305) for patients on ts/bDMARDs and $29 \%$ (35 out of 121$)$ treated with csDMARDs $(\mathrm{p}=0.26)$. By diagnosis, patients treated with ts/bDMARDs who required hospitalisation were $44(62 \%)$ RA, $20(28 \%)$ SpA, $3(4 \%)$ SLE and 4 (6\%) other rheumatic diseases, while those who did not need hospitalisation were: 86 (37\%) RA, 113 (48\%) SpA, 10 (4\%) SLE and 25 (11\%) other rheumatic diseases $(p=0.002)$. In patients treated with csDMARDs, the proportion of patients hospitalised and non-hospitalised by diagnosis showed no statistically significant differences $(\mathrm{p}=0.319)$ (table 2). Hospitalised patients on ts/bDMARDs treatment were consistently elderly (61.4 vs 52.8 years old, $\mathrm{p}<0.001)$, showed a higher age at initiation of treatment ( 53.5 vs $47.3, \mathrm{p}<0.001)$, had a higher BMI (82\% vs $65 \%$ overweight/obesity, $\mathrm{p}=0.023$ ) and had more comorbidities $(61 \%$ vs $33 \%, \mathrm{p}<0.001)$ than non-hospitalised. Among the 121 patients with COVID-19 on csDMARDs, hospitalisation occurred as well in elder patients (68.3 vs 50.4years old, $\mathrm{p}<0.001)$, with more comorbidities $(77 \%$ vs $33 \%, \mathrm{p}<0.001)$, including obesity ( $50 \%$ vs $19 \%, \mathrm{p}=0.018)$. When disease activity prior to COVID-19 symptoms was analysed qualitatively, no differences were observed between the proportions of patients in remission, low, moderate or high activity between those who were hospitalised and those who were not $(p=0.569)$ (data not shown).

In the bivariate analysis (table 3), hospitalised patients were significantly older (61.8 vs 52.2 years old, $\mathrm{p}<0.001)$, had a different rheumatic disorder $(\mathrm{p}=0.003)$ being
RA the most common (54 out of $106,51 \%$ ), a longer disease duration (12.9 vs 10.4 years, $\mathrm{p}=0.017)$, started treatment with biologicals at an older age (54.9 vs 47.3 years old, $\mathrm{p}<0001)$ and had more comorbidities included in the Charlson index $(\mathrm{p}<0.001)$, as well as higher BMI (29.3 vs $\left.26.9 \mathrm{~kg} / \mathrm{m}^{2}, \mathrm{p}=0.001\right)$, compared with the nonhospitalised population.

Regarding DMARDs, hospitalised patients differed from non-hospitalised patients in treatment with biologicals $(p=0.001)$, with a lower frequency of use of TNF-i (25\% vs $46 \%)$. The use of tsDMARDs and csDMARDs was not associated with a different risk of hospitalisation, either alone or in combination $(\mathrm{p}=0.429)$ (online supplemental table 1). In contrast, hospitalised patients had been more frequently treated with glucocorticoids, although the mean dose of these agents did not differ between hospitalised and non-hospitalised ones. In terms of COVID-19 symptoms, hospitalised patients showed more frequently fever, cough, dyspnoea, chest pain, abdominal pain, nausea/vomiting and asthaenia while they exhibited less frequently sore throat and anosmia than non-hospitalised.

In the multivariate analysis (table 4), the factors associated with a significantly increased likelihood of hospitalisation were age (OR 1.053, 95\% 1.024 to 1.082), the presence of overweight/obesity (OR 2.222, 95\% CI 1.043 to 4.733 ) or chronic liver disease (OR 5.003, 95\% 1.036 to 24.161), and the presence of nausea (OR 4.379, 95\% CI 1.708 to 11.225 ), fever (OR 6.093, 95\% CI 2.673 to 13.887), asthaenia (OR 2.263, 95\% CI 1.092 to 4.687 ) and dyspnoea (OR 11.515, 95\% CI 5.453 to 24.316). Factors associated with a significantly lower likelihood of hospitalisation were sore throat (OR 0.210 , 95\% CI 0.074 to 0.595 ) and arthralgia (OR $0.183,95 \%$ CI 0.068 to 0.497 ). Neither the type of the rheumatic disease, nor the form of treatment were significantly associated with hospitalisation, except for patients treated with TNF-i that showed a lower risk for hospitalisation (OR 0.032, 95\% CI 0.125 to $0.821, \mathrm{p}=0.018)$. Conversely, patients treated with antiCD20 showed a trend to hospitalisation by COVID-19 but not reaching a significant difference (OR 4.84, 95\% CI 0.86 to $27.24, \mathrm{p}=0.073)$. The discrimination performance of the model was excellent with an AUROC of 0.93 
Table 2 Characteristics of hospitalised and non-hospitalised patients according to use of ts/bDMARDs and/or csDMARDs

\begin{tabular}{|c|c|c|c|c|c|c|}
\hline \multirow[b]{3}{*}{ Variable } & \multicolumn{3}{|l|}{ ts/bDMARDs } & \multicolumn{3}{|l|}{ csDMARDs } \\
\hline & \multirow{2}{*}{$\begin{array}{l}\text { Hospitalised } \\
(n=71)\end{array}$} & \multirow{2}{*}{$\begin{array}{l}\text { Non-hospitalised } \\
(n=234)\end{array}$} & \multirow[b]{2}{*}{$P$ value } & \multirow{2}{*}{$\begin{array}{l}\text { Hospitalised } \\
(n=35)\end{array}$} & \multirow{2}{*}{$\begin{array}{l}\text { Non-hospitalised } \\
(\mathrm{n}=86)\end{array}$} & \multirow[b]{2}{*}{$P$ value } \\
\hline & & & & & & \\
\hline Age (years), median (IQR) & $61.4(50.6-70.8)$ & $52.8(42.9-61.0)$ & $<0.001$ & $68.3(57.1-76.0)$ & $50.4(42.6-61.3)$ & $<0.001$ \\
\hline Sex (male), n (\%) & $23(32)$ & $77(33)$ & 0.936 & $14(40)$ & $19(22)$ & 0.070 \\
\hline Ethnicity, n (\%) & & & 0.422 & & & 0.385 \\
\hline Caucasian & $61(86)$ & $213(91)$ & & $33(94)$ & $75(87)$ & \\
\hline Latin American & $7(10)$ & $16(7)$ & & $1(3)$ & $9(11)$ & \\
\hline Others & $3(4)$ & $5(2)$ & & $1(3)$ & $2(2)$ & \\
\hline Tobacco & & & 0.056 & & & 0.025 \\
\hline Current smoker & $7(10)$ & $29(12)$ & & - & $15(17)$ & \\
\hline Former smoker & $21(29)$ & $39(17)$ & & $13(37)$ & $31(36)$ & \\
\hline Non-smoker & $43(61)$ & $166(71)$ & & $22(63)$ & $40(47)$ & \\
\hline $\mathrm{BMI}$, mean $\pm \mathrm{SD}$ & $28.9 \pm 5.2$ & $27.0 \pm 5.1$ & 0.023 & $30.3 \pm 7.9$ & $26.6 \pm 5.0$ & 0.014 \\
\hline$\geq 30$ obesity, n (\%) & $19(38)$ & $47(28)$ & 0.221 & $10(50)$ & $12(19)$ & 0.018 \\
\hline Overweight/obesity, n (\%) & $49(82)$ & $121(65)$ & 0.023 & $19(76)$ & $41(62)$ & 0.322 \\
\hline COVID-19 diagnosis, n (\%) & & & 0.018 & & & 0.019 \\
\hline Confirmed cases & $58(82)$ & $156(67)$ & & $29(83)$ & $51(59)$ & \\
\hline Suspected cases & $13(18)$ & $78(33)$ & & $6(17)$ & $35(41)$ & \\
\hline Symptomatic COVID-19, n (\%) & $71(100)$ & $200(85)$ & $<0.001$ & $35(100)$ & $76(88)$ & 0.061 \\
\hline \multicolumn{7}{|l|}{ COVID-19 symptoms, n (\%) } \\
\hline Fever & $63(89)$ & $105(45)$ & $<0.001$ & $29(83)$ & $50(58)$ & 0.011 \\
\hline Cough & $54(76)$ & $118(50)$ & $<0.001$ & $28(80)$ & $46(54)$ & 0.008 \\
\hline Headache & $16(22)$ & $64(27)$ & 0.446 & $7(20)$ & $21(24)$ & 0.812 \\
\hline Sore throat & $8(11)$ & $50(21)$ & 0.059 & $1(3)$ & $23(27)$ & 0.002 \\
\hline Dyspnoea & $47(66)$ & $41(17)$ & $<0.001$ & $23(66)$ & $10(12)$ & $<0.001$ \\
\hline Arthralgia & $12(17)$ & $40(17)$ & 0.970 & $4(11)$ & $20(23)$ & 0.208 \\
\hline Myalgia & $16(22)$ & $51(22)$ & 0.895 & $6(17)$ & $23(27)$ & 0.349 \\
\hline Chest pain & $19(27)$ & $21(9)$ & $<0.001$ & $7(20)$ & $6(7)$ & 0.051 \\
\hline Abdominal pain & $9(13)$ & $12(5)$ & 0.056 & $3(9)$ & $3(4)$ & 0.354 \\
\hline Diarrhoea & $17(24)$ & $50(21)$ & 0.627 & $9(26)$ & $12(14)$ & 0.184 \\
\hline Nausea/vomiting & $17(24)$ & $16(7)$ & $<0.001$ & $3(9)$ & $8(9)$ & 0.899 \\
\hline Anosmia & $10(14)$ & $50(21)$ & 0.232 & $3(9)$ & $19(22)$ & 0.080 \\
\hline Ageusia & $9(13)$ & $45(19)$ & 0.286 & $2(6)$ & $13(15)$ & 0.226 \\
\hline Rhinorrhoea & $5(7)$ & $22(9)$ & 0.640 & $1(3)$ & $13(15)$ & 0.065 \\
\hline Asthaenia & $35(49)$ & $66(28)$ & 0.001 & $13(37)$ & $25(29)$ & 0.396 \\
\hline COVID-19 outcomes, n (\%) & & & $<0.001$ & & & $<0.001$ \\
\hline Recovered without sequelae & $55(77)$ & $228(97)$ & & $20(57)$ & $83(97)$ & \\
\hline Recovered with sequelae & $6(9)$ & $6(3)$ & & $7(20)$ & $2(2)$ & \\
\hline Death & $10(14)$ & - & & $8(23)$ & $1(1)$ & \\
\hline ICU admission, n (\%) & $14(20)$ & - & & $7(20)$ & - & \\
\hline Rheumatic disease & & & 0.002 & & & 0.319 \\
\hline $\mathrm{RA}$ & $44(62)$ & $86(37)$ & & $10(29)$ & $14(16)$ & \\
\hline Spondyloarthropathies & $20(28)$ & $113(48)$ & & $10(29)$ & $22(26)$ & \\
\hline $\begin{array}{l}\text { Systemic lupus } \\
\text { erythematosus }\end{array}$ & $3(4)$ & $10(4)$ & & $10(29)$ & $38(44)$ & \\
\hline
\end{tabular}




\begin{tabular}{|c|c|c|c|c|c|c|}
\hline \multirow[b]{3}{*}{ Variable } & \multicolumn{3}{|l|}{ ts/bDMARDs } & \multicolumn{3}{|l|}{ csDMARDs } \\
\hline & \multirow{2}{*}{$\begin{array}{l}\text { Hospitalised } \\
(\mathrm{n}=71)\end{array}$} & \multirow{2}{*}{$\begin{array}{l}\text { Non-hospitalised } \\
(\mathrm{n}=234)\end{array}$} & \multirow[b]{2}{*}{$P$ value } & \multirow{2}{*}{$\begin{array}{l}\text { Hospitalised } \\
(\mathrm{n}=35) \\
\end{array}$} & \multirow{2}{*}{$\begin{array}{l}\text { Non-hospitalised } \\
(\mathrm{n}=86)\end{array}$} & \multirow[b]{2}{*}{$P$ value } \\
\hline & & & & & & \\
\hline Other rheumatic diseases & $4(6)$ & $25(11)$ & & $5(13)$ & $12(14)$ & \\
\hline $\begin{array}{l}\text { Disease duration (years), } \\
\text { median (IQR) }\end{array}$ & $12.2(6.0-18.3)$ & $10.1(5.4-15.2)$ & 0.165 & $14.6(10.6-22.5)$ & $10.8(5.0-18.5)$ & 0.053 \\
\hline $\begin{array}{l}\text { Age (years) at the initiation of } \\
\text { biologicals, median (IQR) }\end{array}$ & $53.5(43.3-60.1)$ & $47.3(37.8-53.9)$ & $<0.001$ & - & - & \\
\hline $\begin{array}{l}\text { Time (years) from diagnosis to } \\
\text { the first biological median (IQR) }\end{array}$ & $3.9(1.1-10.0)$ & $3.2(1.0-7.3)$ & 0.399 & - & - & \\
\hline $\begin{array}{l}\text { Last available DAS- } 28 \text { in } \\
\text { patients with } R A, \text { mean } \pm S D\end{array}$ & $3.4 \pm 1.6$ & $3.1 \pm 1.7$ & 0.224 & $4.6 \pm 1.4$ & $2.8 \pm 1.4$ & 0.002 \\
\hline \multicolumn{7}{|l|}{ Treatments } \\
\hline Glucocorticoids, n (\%) & $39(55)$ & $65(28)$ & $<0.001$ & $19(54)$ & $28(33)$ & 0.039 \\
\hline $\begin{array}{l}\text { Dose of glucocorticoids } \\
\text { (prior to COVID-19), } \\
\text { mean } \pm \text { SD }\end{array}$ & $6.0 \pm 3.7$ & $6.2 \pm 4.7$ & 0.851 & $5.9 \pm 1.9$ & $8.0 \pm 7.1$ & 0.429 \\
\hline $\begin{array}{l}\text { Dose of glucocorticoids } \\
\geq 10 \mathrm{mg}, \mathrm{n}(\%)\end{array}$ & $6(15)$ & $9(14)$ & 0.829 & $3(16)$ & $5(18)$ & 0.853 \\
\hline NSAIDs, n (\%) & $19(27)$ & $58(25)$ & 0.756 & $7(20)$ & $18(21)$ & 0.909 \\
\hline ACEI, n (\%) & $12(17)$ & $19(8)$ & 0.043 & $3(9)$ & $11(13)$ & 0.755 \\
\hline Comorbidity, n (\%) & $43(61)$ & $77(33)$ & $<0.001$ & $27(77)$ & $28(33)$ & $<0.001$ \\
\hline COPD & $5(7)$ & $2(1)$ & 0.009 & $6(17)$ & 1 (1) & 0.002 \\
\hline Diabetes & $16(22)$ & $21(9)$ & 0.006 & 7 (20) & $8(9)$ & 0.130 \\
\hline Hypertension & $32(45)$ & $62(26)$ & 0.005 & $20(57)$ & $22(26)$ & 0.001 \\
\hline Neoplasm & $5(7)$ & $6(3)$ & 0.136 & $4(11)$ & $3(4)$ & 0.106 \\
\hline Chronic liver disease & $6(9)$ & $8(3)$ & 0.101 & $3(9)$ & $2(2)$ & 0.145 \\
\hline Renal failure & $3(4)$ & $3(1)$ & 0.141 & $4(11)$ & $1(1)$ & 0.024 \\
\hline
\end{tabular}

ACEI, ACE inhibitor; bDMARD, biological disease-modifying antirheumatic drugs; BMI, body mass index; COPD, chronic pulmonary obstructive disease; csDMARD, conventional disease-modifying antirheumatic drugs; ICU, intensive care unit; NSAIDs, non-steroidal antiinflammatory drugs; RA, rheumatoid arthritis; tsDMARD, targeted synthetic disease-modifying antirheumatic drugs.

(95\% CI 0.90 to $0.95 ; \mathrm{p}<0.001$; figure 2 ). Online supplemental table 2 shows the kappa coefficient of concordance between observed and model-predicted data for hospitalised and non-hospitalised patients analysed separately for confirmed (positive test) and suspected (no test performed) COVID-19 cases. The concordance was 0.680 and 0.477 , for confirmed and suspected COVID-19, respectively.

\section{DISCUSSION}

The most important findings of our work can be summarised as follows: (1) in patients with RMDs suffering from COVID-19, the use of bDMARDs or tsDMARDs is not associated with the severity of the viral infection, except for anti-CD20, which showed a tendency to increase the risk of hospitalisation; (2) conversely, the use of TNF-i is associated with a reduction of that risk; (3) demographic and clinical factors associated with a higher probability of hospitalisation for COVID-19 in RMD patients are age, male gender, comorbidities such as obesity and chronic liver disease and the presence of some COVID-19 symptoms such as nausea, fever, asthaenia and dyspnoea; (4) other COVID-19 symptoms, such as sore throat and arthralgias, predict, however, a lower probability of hospitalisation; and (5) factors related to rheumatic disease, including diagnosis and disease duration, do not appear to be associated with the probability of hospitalisation.

The frequency of hospitalisation among rheumatic patients with symptomatic COVID-19 in our study was $27 \%$, lower than that reported by the largest series of patients with rheumatic disorders and COVID-19 reported so far $(46 \%)^{6}$ and by a series of tertiary hospital in Spain $(44 \%) .{ }^{8}$ However, the frequency of hospitalisation among patients with rheumatic disorders reported in Germany (32\%) $)^{714}$ and Iceland $(33 \%)$ are in the range of our results. In addition, a recent study performing serological testing in unselected patients with various RMDs showed figures $(20 \%)$ in line with our finding. ${ }^{23}$ These studies and ours differ in the time frame of case collection, which may be, at least in part, the reason for these 
Table 3 Bivariate comparison of hospitalised versus non-hospitalised patients according to clinical characteristics, type of DMARD, rheumatic disease and COVID-19 symptoms

\begin{tabular}{|c|c|c|c|}
\hline Variable & Hospitalised $(n=106)$ & Non-hospitalised $(n=320)$ & $P$ value \\
\hline Age (years), median (IQR) & $61.8(54.5-73.7)$ & $52.2(42.8-61.1)$ & $<0.001$ \\
\hline Sex (male), n (\%) & $37(35)$ & $96(30)$ & 0.397 \\
\hline Race, n (\%) & & & 0.671 \\
\hline Caucasian & $94(89)$ & $288(90)$ & \\
\hline Latin American & $8(7)$ & $25(8)$ & \\
\hline Other & $4(4)$ & $7(2)$ & \\
\hline Tobacco, n (\%) & & & 0.032 \\
\hline Current smoker & $7(7)$ & $44(14)$ & \\
\hline Former smoker & $34(32)$ & $70(22)$ & \\
\hline Non-smoker & $65(61)$ & $206(64)$ & \\
\hline $\mathrm{BMI}$, mean $\pm \mathrm{SD}$ & $29.3 \pm 6.1$ & $26.9 \pm 5.0$ & 0.001 \\
\hline$\geq 30$ obesity, $\mathrm{n}(\%)$ & $44(41)$ & $89(28)$ & 0.011 \\
\hline Overweight/obesity, n (\%) & $81(76)$ & $203(63)$ & 0.017 \\
\hline COVID-19 diagnosis, n (\%) & & & 0.001 \\
\hline Confirmed cases & $87(82)$ & $207(65)$ & \\
\hline Suspected cases & $19(18)$ & $113(35)$ & \\
\hline Symptomatic COVID-19, n (\%) & $106(100)$ & $276(86)$ & $<0.001$ \\
\hline \multicolumn{4}{|l|}{ COVID-19 symptoms, n (\%) } \\
\hline Fever & $92(87)$ & $155(48)$ & $<0.001$ \\
\hline Cough & $82(77)$ & $164(51)$ & $<0.001$ \\
\hline Headache & $23(22)$ & $85(27)$ & 0.368 \\
\hline Sore throat & $9(8)$ & $73(23)$ & 0.001 \\
\hline Dyspnoea & $70(66)$ & $51(16)$ & $<0.001$ \\
\hline Arthralgia & $16(15)$ & $60(19)$ & 0.465 \\
\hline Myalgia & $22(21)$ & $74(23)$ & 0.688 \\
\hline Chest pain & $26(25)$ & $27(8)$ & $<0.001$ \\
\hline Abdominal pain & $12(11)$ & $15(5)$ & 0.021 \\
\hline Diarrhoea & $26(24)$ & $62(19)$ & 0.270 \\
\hline Nausea/vomiting & $20(19)$ & $24(7)$ & 0.002 \\
\hline Anosmia & $13(12)$ & $69(22)$ & 0.035 \\
\hline Ageusia & $11(10)$ & $58(18)$ & 0.068 \\
\hline Rhinorrhoea & $6(6)$ & $35(11)$ & 0.110 \\
\hline Asthaenia & $48(45)$ & $91(28)$ & 0.002 \\
\hline COVID-19 outcomes, n (\%) & & & $<0.001$ \\
\hline Recovered without sequelae & $75(71)$ & $311(97)$ & \\
\hline Recovered with sequelae & $13(12)$ & $8(2.5)$ & \\
\hline Death & $18(17)$ & $1(0.3)$ & \\
\hline ICU admission, n (\%) & $21(20)$ & - & $<0.001$ \\
\hline Rheumatic disease & & & 0.003 \\
\hline RA & $54(51)$ & $100(31)$ & \\
\hline Spondyloarthropathies & $30(28)$ & $135(42)$ & \\
\hline Systemic lupus erythematosus & $13(12)$ & $48(15)$ & \\
\hline Other rheumatic diseases & $9(9)$ & $37(12)$ & \\
\hline Disease duration (years), median (IQR) & $12.9(6.9-19.8)$ & $10.4(5.3-16.4)$ & 0.017 \\
\hline
\end{tabular}


Table 3 Continued

\begin{tabular}{|c|c|c|c|}
\hline Variable & Hospitalised $(n=106)$ & Non-hospitalised $(n=320)$ & $P$ value \\
\hline Age (years) at the initiation of biologicals, median (IQR) & $54.9(47.2-63.4)$ & $47.3(37.5-54.7)$ & $<0.001$ \\
\hline $\begin{array}{l}\text { Time (years) from diagnosis to the first biological median } \\
\text { (IQR) }\end{array}$ & $4.1(1.1-9.3)$ & $3.1(1.0-7.4)$ & 0.376 \\
\hline Last available DAS- 28 in patient with $\mathrm{RA}$, mean $\pm \mathrm{SD}$ & $3.6 \pm 1.6$ & $3.1 \pm 1.6$ & 0.073 \\
\hline \multicolumn{4}{|l|}{ Treatments } \\
\hline bDMARD, n (\%) & & & $<0.001$ \\
\hline TNF inhibitors & $27(25)$ & $148(46)$ & \\
\hline IL-17-inhibitors & $5(5)$ & $13(4)$ & \\
\hline IL-1-inhibitor & $2(2)$ & - & \\
\hline Anti-B cells & $10(9)$ & $14(4)$ & \\
\hline Anti-CD20 & $9(8)$ & $6(2)$ & \\
\hline Anti-BAFF & $1(1)$ & $8(2)$ & \\
\hline IL-6 inhibitors & $4(4)$ & $17(5)$ & \\
\hline $\begin{array}{l}\text { Other (T cell activation inhibitor, IL-12/23 inhibitor, } \\
\text { RNKL-RANK inhibitor) }\end{array}$ & $12(11)$ & $15(5)$ & \\
\hline tsDMARD, n (\%) & & & 0.783 \\
\hline Apremilast (inh. PDE4) & $2(2)$ & $5(2)$ & \\
\hline Baricitinib & $7(7)$ & $15(5)$ & \\
\hline Tofacitinib & $5(5)$ & $11(3)$ & \\
\hline \multicolumn{4}{|l|}{ csDMARD, n (\%) } \\
\hline Methotrexate & $28(26)$ & $69(22)$ & 0.349 \\
\hline Chloroquine & $10(9)$ & $36(11)$ & 0.719 \\
\hline Others & $15(14)$ & $39(12)$ & 0.615 \\
\hline Glucocorticoids, n (\%) & $58(55)$ & $93(29)$ & $<0.001$ \\
\hline Dose of glucocorticoids (prior to COVID-19), mean \pm SD & $6.0 \pm 3.4$ & $6.6 \pm 5.2$ & 0.561 \\
\hline Dose of glucocorticoids $\geq 10 \mathrm{mg}, \mathrm{n}(\%)$ & $9(16)$ & $13(14)$ & 0.816 \\
\hline NSAIDs, n (\%) & $26(25)$ & $76(24)$ & 0.896 \\
\hline ACEI, n (\%) & $15(14)$ & $30(9)$ & 0.201 \\
\hline Comorbidity, n (\%) & $70(66)$ & $105(33)$ & $<0.001$ \\
\hline COPD & $11(10)$ & $3(1)$ & $<0.001$ \\
\hline Diabetes & $23(22)$ & $29(9)$ & 0.001 \\
\hline Hypertension & $52(49)$ & $84(26)$ & $<0.001$ \\
\hline Neoplasm & $9(9)$ & $9(3)$ & 0.022 \\
\hline Chronic liver disease & 9 (9) & $10(3)$ & 0.029 \\
\hline Renal failure & $7(7)$ & $4(1)$ & 0.007 \\
\hline
\end{tabular}

ACEI, ACE inhibitors; bDMARD, biological disease-modifying antirheumatic drugs; BMI, body mass index; COPD, chronic pulmonary obstructive disease; csDMARD, conventional synthetic disease-modifying antirheumatic drugs; ICU, intensive care unit; IL, interleukin; NSAIDs, non-steroidal anti-inflammatory drugs; RA, rheumatoid arthritis; TNF, tumour necrosis factor; tsDMARD, targeted synthetic diseasemodifying antirheumatic drugs.

differences, as well as channelling bias in the first months of the pandemic.

We found no association between targeted therapies (ts/bDMARDs) analysed as a whole and the risk of hospitalisation for COVID-19 in patients with inflammatory rheumatic diseases. This is in line with other studies ${ }^{89}$; however, our data suggest a protective effect for TNF-i and a trend towards increased risk of hospitalisation with
anti-CD20. The association between TNF-i and a lower risk of hospitalisation in patients with COVID-19 found in our study (OR $0.32,95 \%$ CI 0.12 to $0.84, p=0.018$ ) has been previously reported. ${ }^{62-26}$ These results indicate that the understandable initial caution against the use of biologicals, including TNF-i, in patients with RMD due to their potential effect increasing the susceptibility or severity of COVID-19 is evidence-based unfounded. An 
Table 4 Logistic regression analysis of the factors associated with hospitalisation in patients with rheumatic disease and COVID-19

\begin{tabular}{|c|c|c|}
\hline Variables & $P$ value & OR $(95 \% \mathrm{Cl})$ \\
\hline \multicolumn{3}{|l|}{$\begin{array}{l}\text { Treatment of the rheumatic } \\
\text { disease }\end{array}$} \\
\hline \multicolumn{3}{|l|}{ Treatment (csDMARDs) } \\
\hline ts/bDMARDs & 0.153 & $0.549(0.242$ to 1.249$)$ \\
\hline \multicolumn{3}{|l|}{ bDMARDs } \\
\hline TNF inhibitors & 0.018 & $0.320(0.125$ to 0.821$)$ \\
\hline IL-17-inhibitors & 0.399 & 0.472 (0.082 to 2.701$)$ \\
\hline Anti-CD20 & 0.073 & 4.849 (0.863 to 27.249$)$ \\
\hline Anti-BAFF & 0.796 & 0.529 (0.004 to 66.128$)$ \\
\hline IL-6 inhibitors & 0.294 & $0.400(0.072$ to 2.217$)$ \\
\hline Others & 0.679 & 0.741 (0.180 to 3.057$)$ \\
\hline tsDMARDs & 0.830 & $0.869(0.241$ to 3.130$)$ \\
\hline Glucocorticoids (no) & 0.164 & 1.692 (0.807 to 3.546$)$ \\
\hline \multicolumn{3}{|l|}{ Demographics } \\
\hline Age & $<0.001$ & 1.053 (1.024 to 1.082$)$ \\
\hline Sex (female) & 0.043 & 2.310 (1.025 to 5.207$)$ \\
\hline \multicolumn{3}{|l|}{ Comorbidities } \\
\hline COPD (no) & 0.054 & 6.639 (0.968 to 45.542$)$ \\
\hline Overweight/obesity (no) & 0.039 & 2.222 (1.043 to 4.733$)$ \\
\hline Chronic liver disease (no) & 0.045 & 5.003 (1.036 to 24.161$)$ \\
\hline \multicolumn{3}{|l|}{ COVID-19 symptoms } \\
\hline Fever (no) & $<0.001$ & 6.093 (2.673 to 13.887$)$ \\
\hline Cough (no) & 0.089 & 1.896 (0.908 to 3.959$)$ \\
\hline Sore throat (no) & 0.003 & $0.210(0.074$ to 0.595$)$ \\
\hline Dyspnoea (no) & $<0.001$ & 11.515 (5.453 to 24.316$)$ \\
\hline Arthralgia (no) & 0.001 & $0.183(0.068$ to 0.497$)$ \\
\hline Nausea (no) & 0.002 & 4.379 (1.708 to 11.225$)$ \\
\hline Asthaenia (no) & 0.028 & 2.263 (1.092 to 4.687$)$ \\
\hline \multicolumn{3}{|l|}{ Disease-related factors } \\
\hline \multicolumn{3}{|l|}{ Diagnosis (RA) } \\
\hline Spondyloarthropathies & 0.508 & 0.719 (0.270 to 1.912$)$ \\
\hline $\begin{array}{l}\text { Systemic lupus } \\
\text { erythematosus }\end{array}$ & 0.176 & 0.401 (0.107 to 1.509$)$ \\
\hline Other rheumatic diseases & 0.257 & 0.465 (0.124 to 1.744$)$ \\
\hline
\end{tabular}

bDMARD, biological disease-modifying antirheumatic drugs; COPD, chronic obstructive pulmonary disease; csDMARD, conventional synthetic disease-modifying antirheumatic drugs; ICU, intensive care unit; IL, interleukin; RA, rheumatoid arthritis; TNF, tumour necrosis factor; tsDMARD, targeted synthetic disease-modifying antirheumatic drugs.

independent comment requires the anti-CD20 therapy. In our study, patients treated with anti-CD20 showed a clear trend to increased risk of hospitalisation by COVID-19, which did not reach statistical significance probably due to sample size (OR 4.84, 95\% CI 0.86 to $27.24, \mathrm{p}=0.073$ ). In an individualised analysis, these patients were neither older nor had more comorbidities than the rest of the patients analysed, but they did have a longer duration of illness $(16.3 \pm 9.05$ vs $11.63 \pm 8.37, \mathrm{p}<0.47)$. Recently,

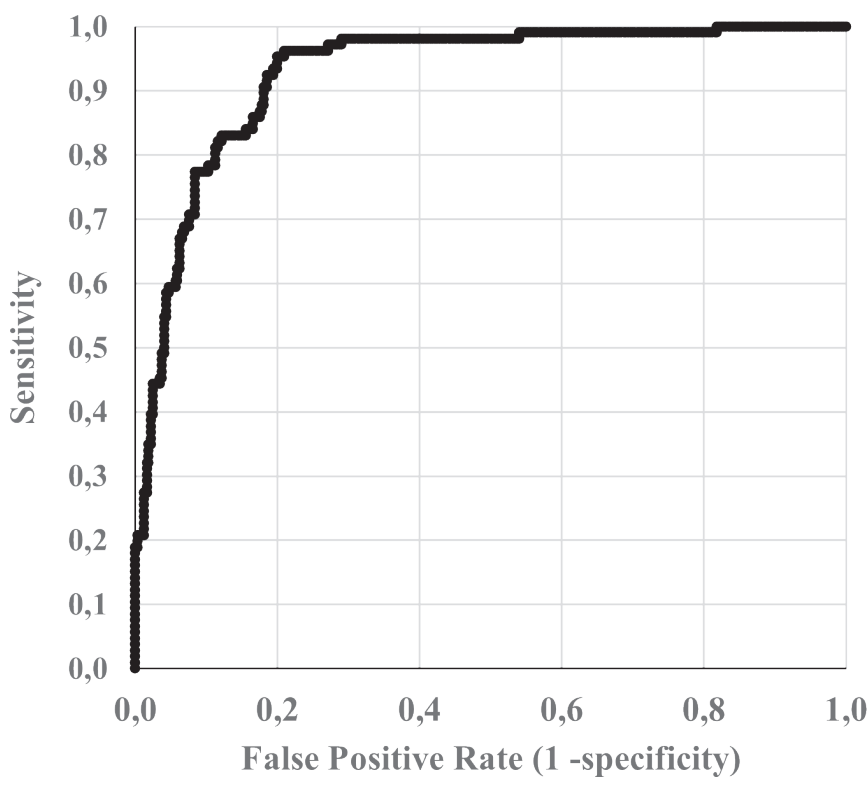

Figure 2 ROC curve for the prediction of hospitalisation according to the logistic regression model. ROC, receiver operating characteristic.

observational studies have described that patients with autoimmune rheumatic diseases treated with this biological had an elevated risk of more severe COVID-19. ${ }^{727}$ Beyond rheumatology, previous studies in multiple sclerosis have found that anti-CD20 agents were associated with a greater risk of developing severe COVID-19-19. ${ }^{28} 29$ These findings suggest that patients with $\mathrm{B}$ cell depleting biologicals should be carefully managed in the context of the current pandemic

Our results did not confirm the role of glucocorticoids by themselves as a factor associated with a greater risk of hospitalisation, despite a positive relationship in the bivariate analysis. This raises the possibility that previous data detecting a greater risk of severe COVID-19 in patients with rheumatic diseases receiving glucocorticoids $^{615}$ could be related to confounding factors like age, disease activity or the severity of the disease. In addition, the fact that glucocorticoids have been shown to be beneficial for severe COVID-19 argues against their effect on hospitalisation. ${ }^{30}$

Of interest, our results did not show a significant effect on hospitalisation of JAK inhibitors despite the reported efficacy of baricitinib and tofacitinib for severe COVID$19 .^{31-34}$ In contrast with some initial warnings on the potential negative effect of the use of NSAIDs or ACE inhibitors, in patients with COVID-19, our data confirm the lack of evidence for these concerns. ${ }^{635} 36$

The demographics, disease-related factors and comorbidities associated with hospitalisation in our study are consistent with those found in previous reports both in rheumatic patients and in the general population. ${ }^{637-41}$ Of interest, our study points at the importance of symptoms of COVID-19 as predictors of hospitalisation (namely, fever, dyspnoea, nausea and asthaenia). We are not aware of other studies that had evaluated this 
potential association in patients with inflammatory rheumatic diseases. In the general population, cough, fever and dyspnoea are among the most common symptoms among hospitalised patients with COVID-19. ${ }^{42} 43$ Some studies have also found a frequent cluster of gastrointestinal symptoms (ie, abdominal pain, vomiting and diarrhoea) among these patients. ${ }^{43}$ It is important to note that although these factors could help to identify patients that require hospitalisation, other factors like older age, male gender, dyspnoea, diabetes and hypertension are better predictors of a higher risk of mortality in the general population $^{44}$ and in patients with rheumatic diseases. ${ }^{15}$ However, several studies indicate that dyspnoea but not fever is associated with a greater risk of mortality. ${ }^{45} 46$ The association between gastrointestinal symptoms and severity of COVID-19 remains to be elucidated. ${ }^{46}$

Consistently with a previous study, ${ }^{6}$ in the adjusted analysis, we did not find that the rheumatic disease diagnosis was associated with a greater likelihood of hospitalisation, suggesting that factors other than the rheumatic disease itself better explain the increased risk of hospitalisation. In contrast, other studies have found greater risks of hospitalisation in patients with systemic autoimmune diseases. ${ }^{810}$ The reasons for these discrepancies are not clear; selection bias can be an important factor in observational studies. However, we believe that the structure of properly designed registries as in our case increases the validity of our findings.

We lacked information on patients' SARS-CoV-2 vaccination status. However, considering that only $6.7 \%$ of the Spanish population had been fully vaccinated by the end of study recruitment, we consider that its impact on the study results is very low.

Strengths of our study include the use of three ongoing, well-established registries in Spain from multiple centres, with a detailed description of demographic and clinical parameters of patients with RMDs that were updated for the COVID-19 features at the onset of the pandemic. These registries follow periodic audits by specialised staff. In addition, the covering of an ample period since the beginning of the pandemic allows for a more realistic and wider approach to the analysis of the impact of COVID-19 in patients with RMDs.

Our study has several limitations as well. Our estimates of the associated factors were restricted by the relatively small number of hospitalisations. In addition, the lack of SARS-CoV-2 confirmatory diagnostic tests in some patients limits the accuracy of the diagnosis, although this scenario was common worldwide in the first months of the pandemic. It is conceivable that some biases like 'recall bias' might exist in relation to the patients' reporting of COVID-19 symptoms, although they were carefully recorded in response to a structured questionnaire. In our work, we consider hospitalisation as the main criterion for severity. However, it is possible that in some specific cases other factors apart from severity (ie, diagnosis, treatments and comorbidities) might have had some role. Although selection bias is a possibility, the structured approach of our study makes it less likely than in other studies where patients were included in the registries just for the fact of developing COVID-19.

In conclusion, our study shows that in patients with autoimmune rheumatic diseases suffering from COVID19 , the use of targeted therapies is not associated with a different prognosis, except for B cell depleting therapy, which showed a tendency to increase the risk of hospitalisation, and TNF-i, which is associated with a reduction of that risk. A mix of sociodemographic factors (age and male gender), comorbidities (obesity/overweight/ obesity and chronic liver disease) and COVID-19 symptoms (dyspnoea, fever, nausea and asthaenia) contribute to an increased risk of hospitalisation of rheumatic patients.

\section{Author affiliations}

${ }^{1}$ Department of Rheumatology, Hospital General Universitario Gregorio Marañón, Madrid, Spain

${ }^{2}$ Research Unit, Sociedad Española de Reumatología, Madrid, Spain

${ }^{3}$ Rheumatology Department, Hospital Universitario Miguel Servet, Zaragoza,

Aragón, Spain

${ }^{4}$ Rheumatology Department, Hospital Universitario Basurto, Bilbao, País Vasco, Spain

${ }^{5}$ Rheumatology Department, Hospital San Rafael, Barcelona, Catalunya, Spain ${ }^{6}$ Department of Rheumatology, Hospital Universitario Príncipe de Asturias, Alcala de Henares, Madrid, Spain

${ }^{7}$ Department of Rheumatology, La Paz University Hospital, Madrid, Spain

${ }^{8}$ Department of Rheumatology, Hospital General Universitario de Alicante, Alicante, Spain

${ }^{9}$ Department of Rheumatology, Hospital Universitario Ramon y Cajal, Madrid, Madrid, Spain

${ }^{10}$ Department of Rheumatology, Hospital Universitario Infanta Leonor, Madrid, Spain

${ }^{11}$ Department of Rheumatology, Hospital Universitario Puerta de Hierro

Majadahonda, Majadahonda, Comunidad de Madrid, Spain

${ }^{12}$ Department of Rheumatology, Hospital Universitario 12 de 0ctubre, Madrid,

Comunidad de Madrid, Spain

${ }^{13}$ Department of Rheumatology, Hospital Doctor Negrin, Las Palmas GC, Spain

${ }^{14}$ Department of Rheumatology, Hospital Universitario Marqués de Valdecilla, Santander, Cantabria, Spain

${ }^{15}$ Department of Rheumatology, University Hospital Complex of Vigo, Vigo, Spain

${ }^{16}$ Departamento de Estadística e Investigación Operativa, Universidad de La Laguna, La Laguna, Islas Canarias, Spain

${ }^{17}$ Department of Rheumatology, Hospital Universitario de Canarias, La Laguna, Spain

${ }^{18}$ Department of Internal Medicine and Psychiatry, Universidad de La Laguna, La Laguna, Spain

\section{Twitter Jose M Pego-Reigosa @PegoReigosa}

Acknowledgements The authors of this work are indebted to all researchers that participate in the BIOBADASER, RELESSER and CARMA projects.

Contributors Conception and design: FD-G, JMAG and CS-P. Analysis and interpretation of data: FD-G, JMAG, CS-P, JTS-C and EG-D. Acquisition of data analysis and interpretation of data: JMP-R, MER-L, LL-V, CB, JM-B, GB, MJG-V, MTN-M, MP, MG, CE, MAG-G, IR-F, JMP-R and ICF. FD-G acted as guarantor. All authors revised the manuscript, approved the final version and were involved in the decision to submit the manuscript for publication to Annals of the Rheumatic Diseases.

Funding This Project has been financed by Bristol-Myers Squibb, Galapagos Biopharma Spain SLU, Gebro Pharma, Roche Farma and Sanofi Aventis.

Competing interests LL-V declares the following conflicts of interest for payment or honoraria for lectures, speakers' bureaus for Lilly. JMAG declares consulting/ lecture/speaker's bureau fees from Abbvie, BMS, Galapagos, Lilly, MSD, Pfizer, Roche and UCB. JMP-R reports the following conflicts of interest for payment or honoraria for lectures, presentations, speakers' bureaus, manuscript writing or educational events from Janssen and Roche. MAG-G received grants/research 
support from AbbVie, MSD, Janssen and Roche and had consultation fees/ participation in company sponsored speaker's bureau from AbbVie, Pfizer, Roche, Celgene, MSD, Novartis, SOBI and Sanofi. FD-G reports the following conflicts of interest for payment or honoraria for lectures, presentations, speakers' bureaus for Lilly, Pfizer, UCB and Novartis; payment for expert testimony for Abbvie, Roche and MSD; support for attending meetings for Pfizer; participation on advisory board for Gilead and Receipt of equipment Abbvie. CE declares support for attending meetings and/or travel from Pfizer and UCB. PV reports receiving consulting fees from Lilly, Novartis and Fresenius Kabi; payment or honoraria for lectures, presentations, speakers' bureaus, manuscript writing or educational events from Sandoz, BMS, GSK, Sanofi and Roche; support for attending meetings and/or travel from Abbvie, Pfizer and Roche and grants or contracts from any entity for her hospital from Abbvie, Roche and MSD. JM-B reports the following conflicts of interest for payment or honoraria for lectures, presentations, speakers' bureaus for UCB, GSK, Lilly and Novartis; support for attending meetings for UCB, GSK and Pfizer; participation on advisory board for UCB and GSK. MG declares the following conflicts of interest for payment or honoraria for lectures, presentations, speakers bureaus for UCB, GSK, Lilly and Abbvie; payment for expert testimony for GSK and participation on advisory board for GSK, Abbvie and UCB.

Patient consent for publication Not applicable.

Ethics approval After consultancy, the Research Ethics Institutions involved in BIOBADASER (CEI/CEIM Hospital Universitari Clinic i Provincial, Barcelona) and in CARMA (Comité de ética da investigación con medicamentos de Galicia) considered that the additional data collection on COVID-19 was a non-relevant modification, and therefore, no new approval was required. For RELESSER-PROS, the Research Ethics Committee (CEI/CEIM Hospital Universitario de Gran Canaria Dr. Negrín approved the modification (Approval code: 130082).

Provenance and peer review Not commissioned; externally peer reviewed.

Data availability statement Data are available on reasonable request. The data supporting the results of this study are the property of the Spanish Society of Rheumatology (SER), and are not publicly available. However, the data are released upon reasoned request and with the permission of the Spanish Society of Rheumatology.

Open access This is an open access article distributed in accordance with the Creative Commons Attribution Non Commercial (CC BY-NC 4.0) license, which permits others to distribute, remix, adapt, build upon this work non-commercially, and license their derivative works on different terms, provided the original work is properly cited, appropriate credit is given, any changes made indicated, and the use is non-commercial. See: http://creativecommons.org/licenses/by-nc/4.0/.

ORCID iDs

Miguel A Gonzalez-Gay http://orcid.org/0000-0002-7924-7406

Federico Diaz-Gonzalez http://orcid.org/0000-0002-4139-9295

\section{REFERENCES}

1 Singh JA, Cameron C, Noorbaloochi S, et al. Risk of serious infection in biological treatment of patients with rheumatoid arthritis: a systematic review and meta-analysis. Lancet 2015;386:258-65.

2 Pérez-Sola MJ, Torre-Cisneros J, Pérez-Zafrilla B, et al. Infections in patients treated with tumor necrosis factor antagonists: incidence, etiology and mortality in the BIOBADASER registry. Med Clin 2011;137:533-40.

3 Mehta P, McAuley DF, Brown M, et al. COVID-19: consider cytokine storm syndromes and immunosuppression. Lancet 2020;395:1033-4.

4 Putman M, Chock YPE, Tam H, et al. Antirheumatic disease therapies for the treatment of COVID-19: a systematic review and meta-analysis. Arthritis Rheumatol 2021;73:36-47.

5 RECOVERY Collaborative Group. Tocilizumab in patients admitted to hospital with COVID-19 (recovery): a randomised, controlled, openlabel, platform trial. Lancet 2021;397:1637-45.

6 Gianfrancesco M, Hyrich KL, Al-Adely S, et al. Characteristics associated with hospitalisation for COVID-19 in people with rheumatic disease: data from the COVID-19 global rheumatology alliance physician-reported registry. Ann Rheum Dis 2020;79:859-66.

7 Hasseli R, Mueller-Ladner U, Hoyer BF, et al. Older age, comorbidity, glucocorticoid use and disease activity are risk factors for COVID-19 hospitalisation in patients with inflammatory rheumatic and musculoskeletal diseases. RMD Open 2021;7:e001464.

8 Freites Nuñez DD, Leon L, Mucientes A, et al. Risk factors for hospital admissions related to COVID-19 in patients with autoimmune inflammatory rheumatic diseases. Ann Rheum Dis 2020;79:1393-9.
9 Pablos JL, Galindo M, Carmona L, et al. Clinical outcomes of hospitalised patients with COVID-19 and chronic inflammatory and autoimmune rheumatic diseases: a multicentric matched cohort study. Ann Rheum Dis 2020;79:1544-9.

10 Pablos JL, Abasolo L, Alvaro-Gracia JM, et al. Prevalence of hospital PCR-confirmed COVID-19 cases in patients with chronic inflammatory and autoimmune rheumatic diseases. Ann Rheum Dis 2020;79:1170-3.

11 Akiyama S, Hamdeh S, Micic D, et al. Prevalence and clinical outcomes of COVID-19 in patients with autoimmune diseases: a systematic review and meta-analysis. Ann Rheum Dis 2021;80:384-91.

12 D'Silva KM, Serling-Boyd N, Wallwork R, et al. Clinical characteristics and outcomes of patients with coronavirus disease 2019 (COVID-19) and rheumatic disease: a comparative cohort study from a US 'hot spot'. Ann Rheum Dis 2020;79:1156-62.

13 Sarmiento-Monroy JC, Espinosa G, Londoño M-C, et al. A multidisciplinary registry of patients with autoimmune and immunemediated diseases with symptomatic COVID-19 from a single center. $J$ Autoimmun 2021;117:102580.

14 Bjornsson AH, Grondal G, Kristjansson M, et al. Prevalence, admission rates and hypoxia due to COVID-19 in patients with rheumatic disorders treated with targeted synthetic or biologic disease modifying antirheumatic drugs or methotrexate: a nationwide study from Iceland. Ann Rheum Dis 2021;80:671-2.

15 Strangfeld A, Schäfer M, Gianfrancesco MA, et al. Factors associated with COVID-19-related death in people with rheumatic diseases: results from the COVID-19 global rheumatology alliance physician-reported registry. Ann Rheum Dis 2021;80:930-42.

16 Brenner EJ, Ungaro RC, Gearry RB, et al. Corticosteroids, but not TNF antagonists, are associated with adverse COVID-19 outcomes in patients with inflammatory bowel diseases: results from an international registry. Gastroenterology 2020;159:481-91. e483.

17 Valenzuela-Almada MO, Putman MS, Duarte-García A. The protective effect of rheumatic disease agents in COVID-19. Best Pract Res Clin Rheumatol 2021;35:101659.

18 Monti S, Balduzzi S, Delvino P, et al. Clinical course of COVID-19 in a series of patients with chronic arthritis treated with immunosuppressive targeted therapies. Ann Rheum Dis 2020;79:667-8.

19 Hyrich KL, Machado PM. Rheumatic disease and COVID-19: epidemiology and outcomes. Nat Rev Rheumatol 2021;17:71-2.

20 Sanchez-Piedra C, Hernández Miguel MV, Manero J, et al. Objectives and methodology of BIOBADASER phase III. Reumatol Clin 2019;15:229-36.

21 Castañeda S, Martín-Martínez MA, González-Juanatey C, et al. Cardiovascular morbidity and associated risk factors in Spanish patients with chronic inflammatory rheumatic diseases attending rheumatology clinics: baseline data of the CARMA project. Semin Arthritis Rheum 2015;44:618-26.

22 Fernández-Nebro A, Rúa-Figueroa Înigo, López-Longo FJ, et al. Cardiovascular events in systemic lupus erythematosus: a nationwide study in Spain from the RELESSER registry. Medicine 2015;94:e1183.

23 Saadoun D, Vieira M, Vautier M, et al. SARS-CoV-2 outbreak in immune-mediated inflammatory diseases: the Euro-COVIMID multicentre cross-sectional study. Lancet Rheumatol 2021;3:e481-8.

24 Veenstra J, Buechler CR, Robinson G, et al. Antecedent immunosuppressive therapy for immune-mediated inflammatory diseases in the setting of a COVID-19 outbreak. J Am Acad Dermatol 2020;83:1696-703.

25 Marques CDL, Kakehasi AM, Pinheiro MM, et al. High levels of immunosuppression are related to unfavourable outcomes in hospitalised patients with rheumatic diseases and COVID-19: first results of ReumaCoV Brasil registry. RMD Open 2021;7:e001461.

$26 \mathrm{FAI} 2 \mathrm{R} / \mathrm{SFR} / \mathrm{SNFMI/SOFREMIP/CRI/IMIDIATE} \mathrm{consortium} \mathrm{and}$ contributors. Severity of COVID-19 and survival in patients with rheumatic and inflammatory diseases: data from the French RMD COVID-19 cohort of 694 patients. Ann Rheum Dis 2020. doi:10.1136/annrheumdis-2020-218310. [Epub ahead of print: 02 Dec 2020].

27 Avouac J, Drumez E, Hachulla E, et al. COVID-19 outcomes in patients with inflammatory rheumatic and musculoskeletal diseases treated with rituximab: a cohort study. Lancet Rheumatol 2021;3:e419-26.

28 Safavi F, Nourbakhsh B, Azimi AR. B-cell depleting therapies may affect susceptibility to acute respiratory illness among patients with multiple sclerosis during the early COVID-19 epidemic in Iran. Mult Scler Relat Disord 2020;43:102195. 
29 Sormani MP, De Rossi N, Schiavetti I, et al. Disease-Modifying therapies and coronavirus disease 2019 severity in multiple sclerosis. Ann Neurol 2021;89:780-9.

30 , Sterne JAC, Murthy S, et al, WHO Rapid Evidence Appraisal for COVID-19 Therapies (REACT) Working Group. Association between administration of systemic corticosteroids and mortality among critically ill patients with COVID-19: a meta-analysis. JAMA 2020;324:1330-41.

31 Goletti D, Cantini F. Baricitinib Therapy in Covid-19 Pneumonia - An Unmet Need Fulfilled. N Engl J Med 2021;384:867-9.

32 Fact sheet for healthcare providers emergency use Authorization (EUA) of BARICITINIB, 2021. Available: https://www.fda.gov/media/ 143823/download

33 Guimarães PO, Quirk D, Furtado $\mathrm{RH}$, et al. Tofacitinib in patients hospitalized with Covid-19 pneumonia. N Engl J Med 2021;385:406-15.

34 Day M. Covid-19: European drugs agency to review safety of ibuprofen. BMJ 2020;368:m1168.

35 Baral R, Tsampasian V, Debski M, et al. Association between reninangiotensin-aldosterone system inhibitors and clinical outcomes in patients with COVID-19: a systematic review and meta-analysis. JAMA Netw Open 2021;4:e213594.

36 Petrilli CM, Jones SA, Yang J, et al. Factors associated with hospital admission and critical illness among 5279 people with coronavirus disease 2019 in New York City: prospective cohort study. BMJ 2020;369:m1966.

37 Price-Haywood EG, Burton J, Fort D, et al. Hospitalization and mortality among black patients and white patients with Covid-19. N Engl J Med 2020;382:2534-43.
38 Alaa A, Qian Z, Rashbass J, et al. Retrospective cohort study of admission timing and mortality following COVID-19 infection in England. BMJ Open 2020;10:e042712.

39 Richardson S, Hirsch JS, Narasimhan M, et al. Presenting characteristics, comorbidities, and outcomes among 5700 patients hospitalized with COVID-19 in the new York City area. JAMA 2020;323:2052-9.

40 Gallo Marin B, Aghagoli G, Lavine K, et al. Predictors of COVID-19 severity: a literature review. Rev Med Virol 2021;31:1-10.

41 International Severe Acute Respiratory and Emerging Infections Consortium (ISARIC). COVID-19 report: 19 May 2020, 2020.

42 Docherty AB, Harrison EM, Green CA, et al. Features of 20133 UK patients in hospital with covid-19 using the ISARIC WHO Clinica Characterisation Protocol: prospective observational cohort study. BMJ 2020;369:m1985.

43 Chidambaram V, Tun NL, Haque WZ, et al. Factors associated with disease severity and mortality among patients with COVID-19: a systematic review and meta-analysis. PLoS One 2020;15:e0241541.

44 Zheng Z, Peng F, Xu B, et al. Risk factors of critical \& mortal COVID-19 cases: A systematic literature review and meta-analysis. $J$ Infect 2020;81:e16-25.

45 Shi L, Wang Y, Wang Y, et al. Dyspnea rather than fever is a risk factor for predicting mortality in patients with COVID-19. J Infect 2020;81:647-79.

46 Zhang Y, Ma P, Zhang X, et al. Association of digestive symptoms with severity and mortality of COVID-19: a protocol for systematic review and meta-analysis. Medicine 2020;99:e22736. 